\title{
The PADME experiment
}

\section{Gabriele Chiodini*, On behalf of the PADME collaboration. ${ }^{\dagger}$}

INFN sezione di Lecce, via Arnesano 73100 Lecce - Italy.

E-mail: gabriele.chiodini@le.infn.it

PADME is a fixed target experiment designed to search for a hypothetical dark photon A' produced in positron-electron annihilations by using a $550 \mathrm{MeV}$ bunched positron beam. It is expected to be sensitive to the parameter $\varepsilon$, describing the mixing between $\mathrm{A}^{\prime}$ and the photon, for $\varepsilon>10^{-4}$ and for values of the $\mathrm{A}^{\prime}$ mass $\mathrm{m}_{A^{\prime}} \leq 23.7 \mathrm{MeV} / \mathrm{c}^{2}$ after about one year of running.

The PADME experiment searches for a dark photon by the missing mass technique in events with only one photon in the final state. In case of discovery, this technique has the advantage to be not controversial, being a bump above a continuous background of standard electromagnetic processes.

An excellent missing mass resolution is obtained with a high resolution BGO calorimeter, which is further improved by using a narrow positron beam and an active target to determine the beam position bunch-by-bunch.

An other crucial aspect of PADME is the use of a bunched positron beam to increase the number of positron on target (POT) and improve the search sensitivity. This choice leads to an increase of events occurring in the same positron bunch which must be disentangled. The strategy to cope with pile-up events foresees to use fast detectors, to veto on charged particles and small angle photons, to digitize all detector waveforms, by a powerful data acquisition, and to develop robust multi-hit reconstruction algorithms.

This paper illustrates the status of the PADME experiment installed at the Beam Test Facility of Laboratori Nazionali di Frascati after the conclusion of the first data taking period from September 2018 to February 2019.

European Physical Society Conference on High Energy Physics - EPS-HEP2019 -

10-17 July, 2019

Ghent, Belgium

\footnotetext{
* Speaker.

${ }^{\dagger}$ Padme collaboration: P. Albicocco, F. Bossi, B. Buonomo, R. De Sangro, D. Domenici, G. Finocchiaro, L.G. Foggetta, A. Ghigo, F. Giacchino, P. Gianotti, I. Sarra, B. Sciascia, T. Spadaro, E. Spiriti, E. Vilucchi (INFN Laboratori Nazionali di Frascati), A.P. Caricato, F. Gontad, M. Martino, I. Oceano, F. Oliva, S.Spagnolo (INFN Sezione di Lecce and Dip. di Matematica e Fisica, Universitá del Salento), C. Cesarotti, A. Frankenthal, J. Alexander (Department of Physics, Cornell University), G. Chiodini (INFN Sezione di Lecce), F. Ferrarotto, E. Leonardi, F. Safai Tehrani, P. Valente (INFN Sezione di Roma), S. Fiore (INFN Sezione di Roma and ENEA), G. Georgiev (University of Sofia St. K1. Ohridski, INFN Laboratori Nazionali di Frascati), V. Kozhuharov (University of Sofia St. Kl. Ohridski, INFN Laboratori Nazionali di Frascati, and Dip. di Fisica, Sapienza Universitá di Roma), B. Liberti, C. Taruggi (INFN Laboratori Nazionali di Frascati and Universitá degli Studi di Roma Tor Vergata), G.C.Organtini, G. Piperno, M.Raggi (INFN Sezione di Roma e Dip. di Fisica, Sapienza - Universitá di Roma), L. Tsankov (University of Sofia St. Kl. Ohridski).
} 


\section{Introduction}

Observation of anomalous gravitational effects in astronomy is not only a strong indication of the existence of dark matter but also that its density dominates the matter density of the Universe [1]. The rich particle structure of the visible sector naturally induce to speculates on a similar behaviour for the dark sector and dark photons could be one of the simplest mediators of interactions among dark matter particles [2]. In addition, dark photons could explain several observed anomalies in astrophysics (e.g. in cosmic rays [3]), that could be related to dark matter interacting with a dark photon, and, most interesting, to explain the discrepancy between the measured and the calculated anomalous magnetic moment of the muon [4].

A massive dark photon A' could be related to a new abelian broken gauge symmetry U(1)' and interactions with ordinary matter can happen only through kinetic mixing $\frac{\varepsilon}{2} F_{\mu \nu} F^{\prime \mu \nu}$ with the photon. This corresponds to an effective interaction $\alpha^{\prime}=\varepsilon^{2} \alpha_{e m}$ with SM particles likely suppressed by $\varepsilon^{2}$ with respect to interaction of invisible particles carrying $\mathrm{U}(1)$ ' quantum numbers. The smallness of $\varepsilon$ would explain why the dark photon escaped so far detection. The search for dark photons can be pursued with low-cost, high-impact experiments at high intensity accelerators or electromagnetic radiation sources.

PADME searches for a dark photon produced in positron-electron annihilation events (Figure 1a) in the interaction between a positron beam and a target [5]. The search technique is based on measuring the missing mass of events with only one photon in the final state, which has the advantage to be independent of the final state (visible $\mathrm{A}^{\prime} \rightarrow e^{+} e^{-}$or invisible $\mathrm{A}^{\prime} \rightarrow \chi \bar{\chi}$, as shown in Figure 1a) and is not controversial in case of discovery, showing a bump above a continuous background. The most abundant background is from Bremsstrahlung events (Figure 1b), where the scattered positron and nuclei are not detected, followed by two or three photons annihilation events (Figure 1c-d) where one or two photons are escaping the experiment acceptance.

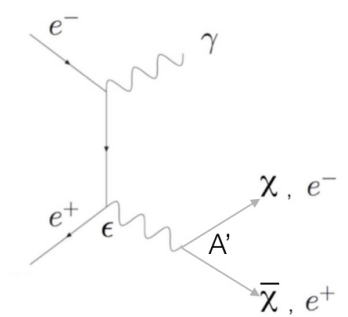

a)

Figure 1: Production mechanisms, by positron-electron annihilation, of a dark photon in association with one photon (a), of two (c) and three (d) photons, and production mechanism of a photon by positron Bremsstrahlung (b). The positron Bremsstrahlung is the main background to the dark photon signal.

The experiment uses an active diamond target, which also monitors each positron bunch, and a dipole field, which bends the charged particles outside the acceptance of the calorimeter system. The calorimeter system is composed of a high resolution electromagnetic calorimeter, made by BGO crystals, to measure photons energy and direction, and of a small angle fast electromagnetic calorimeter, made of $\mathrm{PbF}_{2}$ crystals, to veto small angle photons. In addition, a charged particle veto 
system, made of vertical bars of fast plastic scintillators, allows to identify electrons and positrons and measure their momentum. Finally, a data acquisition system is capable to store all the frontend waveforms for each detector event-by-event and preserves all the information, for multi-hit reconstruction and time resolution optimization.

\section{The positron beams at the Beam Test Facility}

The PADME experiment is located in the first experimental hall of the new Beam Test Facility (BTF) [6] of the DA $\Phi N E$ accelerator complex at Laboratori Nazionali di Frascati (LNF). Electrons or positrons can be extracted before the injection into the damping ring (see Fig. 2), on a bunchby-bunch basis, by means of a pulsed dipole and driven to the BTF by a dedicated transfer line made of 2 FODO quadrupoles doublets for focussing. With the new electron gun system, pulses as long as $280 \mathrm{~ns}$ have been successfully accelerated with about $1 \%$ energy spread, $1.5 \mathrm{~mm}$ beam spot, $1.5 \mathrm{mrad}$ emittance at the rate of $50 \mathrm{~Hz}$. Positrons can be accelerated up to $550 \mathrm{MeV}$ after being generated in the LINAC with a W-Re converter of $2 \mathrm{X}_{0}$ located after the first sections, where electrons have been accelerated up to $220 \mathrm{MeV}$ (primary positron beam). Alternatively, a secondary positron beam of slightly higher energy can be generated by a primary electron beam of $750 \mathrm{MeV}$ hitting a $\mathrm{Cu}$ converter of selectable $\mathrm{X}_{0}=1.7,2$, and 2.3 (BTF target) located before $1 \mathrm{~m}$ thick BTF concrete wall. An energy selection system and collimators on the BTF transfer-line defines momentum, spot size, and intensity.

The PADME experiment started commissioning on September 2018 and took data until the end of February 2019 with a bunch multiplicity of about $20000 \div 30000$ positrons and up to $250 \mathrm{nsec}$ length. The collected data correspond to about $7.5 \times 10^{12}$ POT, mostly taken with the secondary positron beam and only a small fraction with the primary positron beam. The data were very useful to setup the beam line, to calibrate the detectors, and to establish the running conditions for the real physics run expected in 2020. The on-going data analysis clearly shows the need to reduce the observed beam background, likely due to non-gaussian tails hitting the beam line and the target frame.

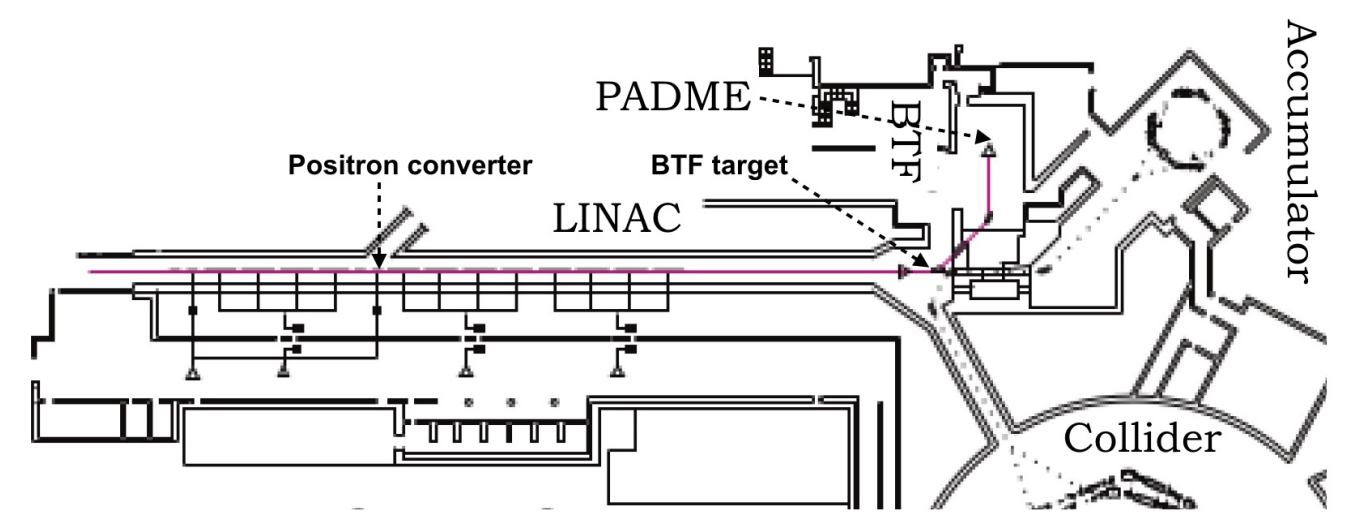

Figure 2: Layout of the positron production complex at LNF. 


\section{The PADME detector}

In Fig. 3 a schematic drawing of the PADME detector is shown. The PADME beam monitors, made of hybrid and monolithic silicon pixel detectors, and the full containment BTF Cherenkov calorimeter, used for absolute beam calibration, are not shown for simplicity.

A bunched positron beam hits the active diamond target placed in the vacuum transport line and in front of $0.5 \mathrm{~T}$ warm magnetic dipole MBP-S shipped from CERN. The dipole field bends the charged particles outside the acceptance of the calorimeter system, inside the large PADME vacuum chamber $\left(\approx 1 \mathrm{~m}^{3}\right)$.

More specifically, not interacting positrons from the beam are driven in the beam dump (thick grey curved arrow) where hybrid pixel detectors are installed outside the vacuum chamber behind of a $1 \mathrm{~mm}$ thick aluminium flange. Interacting positrons and electrons are bent in the charged particle Veto systems (blue curved arrows) where they are detected as single Minimum Ionizing Particles (MIPs) and their momentum measured accordingly to the arrival position.

Photons produced in the target preserve the initial direction and are detected in the calorimeter system (red straight arrows), which provides information on the energy and direction. The calorimeter system is located outside the vacuum chamber in front of a large and thin carbon fibre flange. It is composed of a high energy resolution electromagnetic calorimeter (ECAL) and a small angle fast electromagnetic calorimeter (SAC) located behind. The ECAL has a hole in the center to avoid the large flux of Bremsstrahlung photons generated in the target by the high multiplicity positron bunch. Instead, the SAC is tailored to handle this flux of photons and is crucial to veto small angle photons from two and three gamma annihilation events, where one photon is inside the ECAL acceptance and in time with, at least, another one inside the SAC acceptance. In fact, dark photon events (yellow arrow) are expected to have only one photon in the final state and no charged particles in time (invisible decay), or two opposite sign charged particle in-time (visible decay).

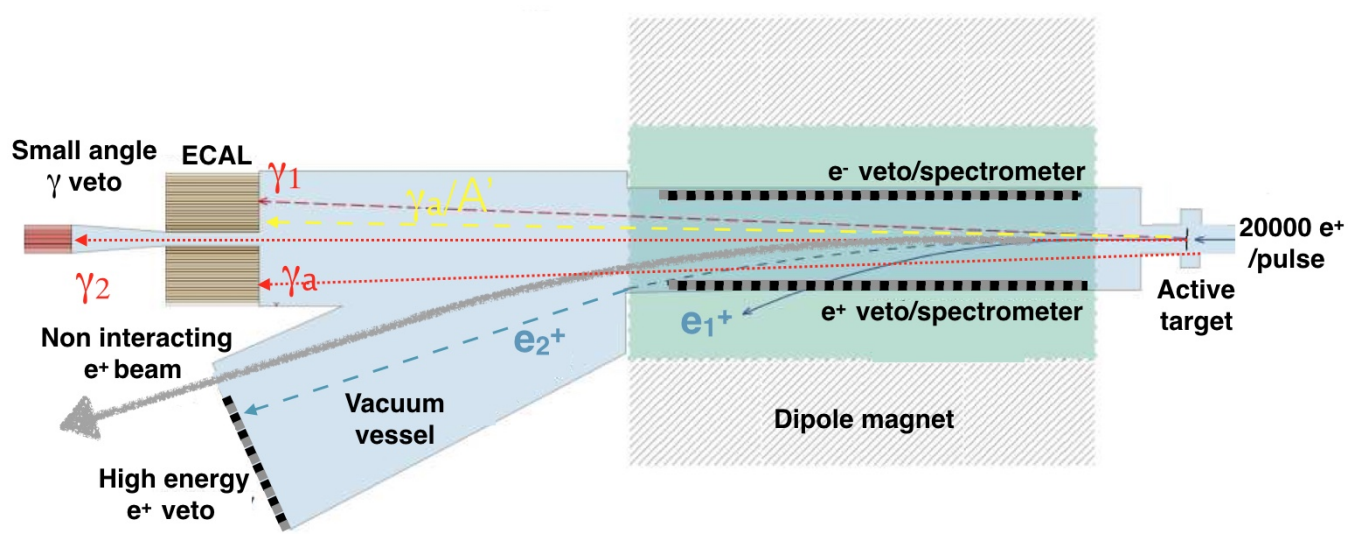

Figure 3: Schematic drawing of the PADME experiment at the BTF of LNF (not to scale).

\subsection{Diamond Active Target}

The target is made of a "full carbon" double sided diamond detector in order to have a small $\mathrm{Z}$ material and to monitor the positron bunches in real time. In fact, the signal cross-section scales as 
$\mathrm{Z}$ and the Bremsstrahlung cross-section as $\mathrm{Z}^{2}$, and a low $\mathrm{Z}$ assures a larger signal-to-background ratio and a smaller event pile-up rate.

The target and the front-end electronics are placed in vacuum but outside the magnetic field (Fig. 4 left). The mechanical structure is in aluminium and can be moved in and out the beam in one direction by a step motor.

The diamond detector is made of an "as grown" CVD polycrystalline diamond sensor of $2 \times 2$ $\mathrm{cm}^{2}$ area and $100 \mu \mathrm{m}$ thickness. On both sensor surfaces 19X+19Y graphite strips were fabricated by an excimer laser with a pitch of $1 \mathrm{~mm}$, an inter-strip distance of $0.15 \mathrm{~mm}$, and an electric resistance of about $2.5 \mathrm{k} \Omega$ [7]. $16 \mathrm{X}+16 \mathrm{Y}$ strips are instrumented by two evaluation boards of the AMADEUS chip made by IDEAS (Oslo). The AMADEUS charge sensitive preamplifiers have a 20-40 ns adjustable shaping time and an adjustable gain from 3 to $24 \mathrm{mV} / \mathrm{fC}$. The measured charge collection distance of the sensor at $250 \mathrm{~V}$ of high voltage bias was about $12 \mu \mathrm{m}$, which corresponds to a signal of about $0.07 \mathrm{fC} / \mathrm{MIP}$.

The active target provided $\mathrm{X}$ and $\mathrm{Y}$ single bunch profiles and the evaluation of the number of POT per bunch performing online beam monitoring and offline luminosity measurement.

\subsection{The charged particle veto system}

The charged particle veto system [8] is composed of three modules all made by polystyrenebased plastic scintillating rods with $1.5 \%$ POPOP produced by UNIPLAST with a $1 \times 1 \mathrm{~cm}^{2}$ crosssection and $17.8 \mathrm{~cm}$ length placed vertically (Fig. 4 center).

The positron veto, the high energy positron veto, and the electron veto are made of 90, 16 and 96 rods, respectively. The rods are slightly rotated $(\approx 0.1 \mathrm{rad})$, to minimize geometrical inefficiencies, and kept in position by an aluminium mounting frame. They are all inside the vacuum chamber but only the positron and the electron vetos are inside the magnetic field.

The scintillating light is collected at one or two ends by BCF-92 wavelength shifter fibers of $1.2 \mathrm{~mm}$ diameter glued into a longitudinal groove. The photons emitted by the fibers are converted into electric signal by S13360 silicon photomultipliers from Hamamatsu. They operate in vacuum and are mounted on the front-end electronics board hosting a group of four custom amplifiers of gain equal four. The boards are powered and managed with custom-built controllers. The achieved time resolution of all scintillator rods, as measured with respect to the small angle calorimeter, is about 700 psec.

\subsection{The electromagnetic calorimeter system}

In Fig. 4 right) a schematic drawing of the two electromagnetic calorimeters of PADME is shown [9]. The ECAL consists of 616 BGO crystals, each of $2.1 \times 2.1 \times 23 \mathrm{~cm}^{3}$ size, arranged in a cylindrical shape of $29 \mathrm{~cm}$ radius with a central square hole of $5 \times 5$ crystals and placed at 3.45 $\mathrm{m}$ from the target. The ECAL angular acceptance is from $15 \mathrm{mrad}$ to $84 \mathrm{mrad}$ and the scintillating light is detected by HZC XP1911 photomultipliers from HZC Photonics. The photomultipliers are directly glued to the painted crystals.

The metal support structure has a square shape and plastic fillers maintain in place each crystal layer. To reduce the light crosstalk, $50 \mu \mathrm{m}$ of black tedlar are inserted between adjacent crystal layers. 
The expected energy resolution on a photon cluster is of the order of $2 \% / \sqrt{E}$ as already obtained in a test with a prototype [10]. The BGO crystal has a high light yield and a fast response but a relatively slow decay time. In fact, $10 \%$ of the BGO scintillating light decays in $60 \mathrm{~ns}$, while the remaining $90 \%$ decays in about $300 \mathrm{~ns}$. This requires an efficient multi-hits reconstruction of signals overlapping in time and an off-line correction to recover the signal tail outside the sampling window of $1 \mu \mathrm{sec}$.

The fast small angle calorimeter (SAC) is placed $50 \mathrm{~cm}$ behind ECAL, in correspondence of the $10.5 \times 10.5 \mathrm{~cm}^{2}$ central hole, where the Bremsstrahlung photon rate is too high for the ECAL. It consists of 25 Cherenkov $\mathrm{PbF}_{2}$ crystals of $3 \times 3 \times 14 \mathrm{~cm}^{3}$ size, which are readout by Hamamatsu R13478UV photomultipliers. Thanks to the fast Cherenkov light signal (about $2.2 \mathrm{~ns}$ for a signal to be completely developed), the pile-up is very low and a single crystal can handle a photon rate of about $300 \mathrm{MHz}$ with an offline multi-hits reconstruction based on a peak finder algorithm.
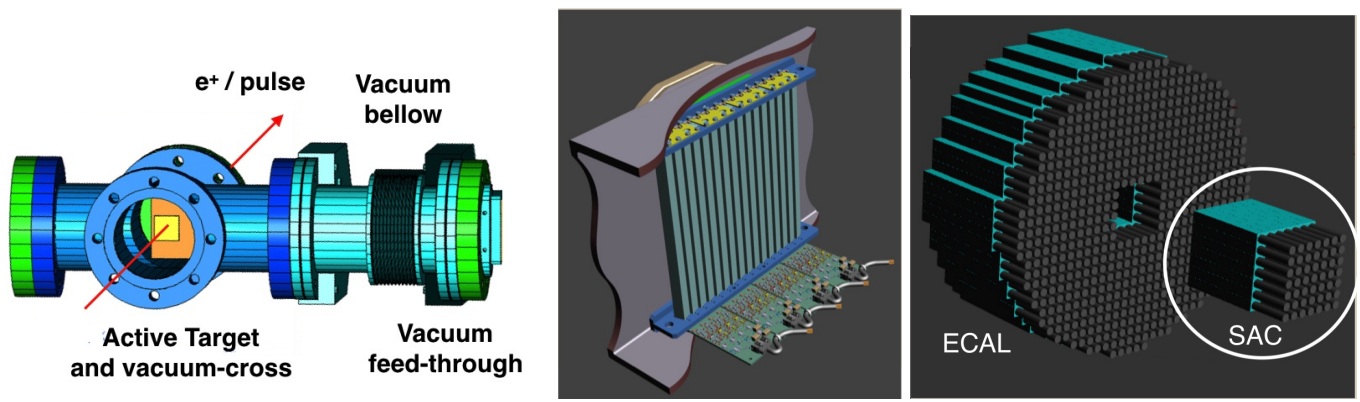

Figure 4: Schematic drawing of: PADME interaction region (left), where the diamond active target is located, PADME positron veto (center), and PADME electromagnetic calorimeter system (right).

\subsection{The data acquisition system}

The PADME data acquisition system is based on the paradigm to digitize and store the waveforms of each detector channel in order to preserve timing information and mitigate the effect of the pile-up by offline signal processing. In Fig. 5 examples of typical waveforms for each detector are shown. The diamond detector signals are integrated on the entire readout window to extract the number of POT per bunch and determine the beam position with a charge-weighting algorithm. The other detectors must reconstruct single physical objects, such as MIPs and photons, with a timing resolution better than $1 \mathrm{nsec}$ and a multi-hit reconstruction algorithm is needed. For the Veto detector and the ECAL, the algorithms must disentangle signals overlapping in time, keeping good efficiency and time resolution, and for the ECAL also good energy resolution.

Approximately 1000 front-end channels are sampled with 12 bit ADC, in a range of $1 \mathrm{~V}$, at a speed of 1-2.5 Gs/s by thirty VME CAEN V1742 digitizer modules, each one equipped with 32 channels.

The trigger signals (Physics, Cosmics, and Random) are generated by the CPU Trigger Board and delivered to all digitizer modules $(2 \times 32$ channels) by the Trigger Distribution Boards. The Physics trigger is synchronous with the BTF clock of $50 \mathrm{~Hz}$ and the Cosmics and Random triggers are asynchronous and interleaved in the data.

The events are further filtered on-line by two Trigger levels: Level 0 servers perform data collection from single boards and zero suppression, while Level 1 servers perform event merging 
and, eventually, a further selection based on full event information. The event data size corresponds to about $900 \mathrm{~KB} /$ bunch and the sustained data throughput is $60 \mathrm{MB} / \mathrm{s}$.
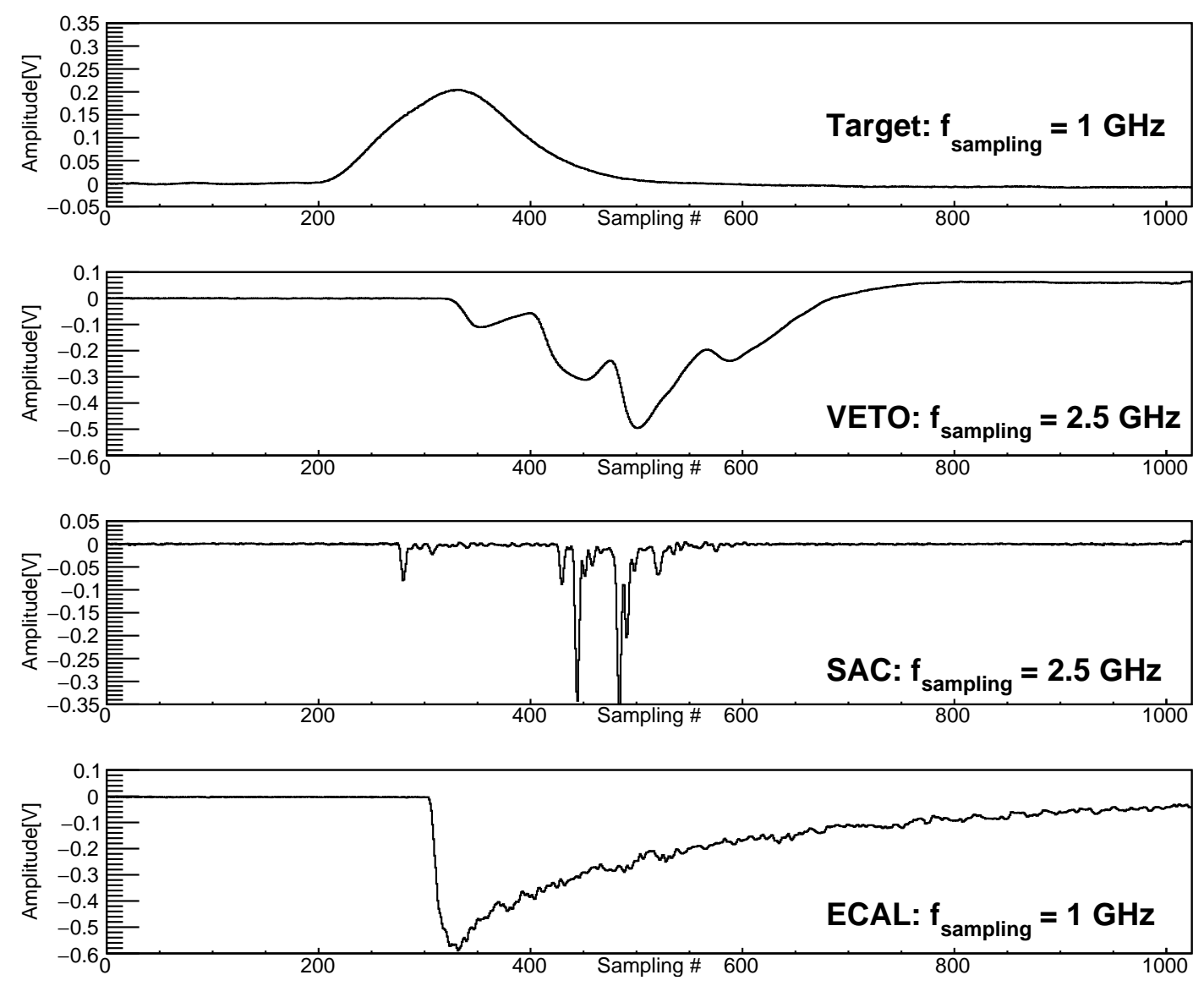

Figure 5: A typical digitized waveform of the active diamond target (a), of the charged particle veto (b), of the SAC (c), and of the BGO calorimeter (d) for a positron bunch of about 20000 positron and $250 \mathrm{nsec}$ time duration. The number of particles crossing the channel varies with the detector: many thousands for the target, four for the charged particle veto, few tens for the SAC, and one for the ECAL.

\section{Conclusions}

PADME is the first experiment to search for a dark photon with the missing mass technique and a pulsed positron beam.

The detector and the data acquisition system reached the design performances and $7.5 \times 10^{12}$ POT were collected with stable positrons beams during the experiment commissioning runs.

The next steps of the collaboration is to finalize the detectors absolute calibration, to measure Bremsstrahlung and annihilation cross-sections with the collected data, minimize the beam background, and, finally, to do a physics run in 2020 to collect up to about $10^{13}$ POT. 


\section{Acknowledgments}

We warmly thank the BTF and LINAC teams of LNF for providing an excellent quality beam and full support during all data taking period. The PADME Target group is also grateful to G. Ambrosi and M. Ionica of INFN Perugia for the wire bonding of the diamond detector. This work is partly supported by the BG-NSF DN-08/14 from 12.2016 and the MoU SU LNF-INFN 70-06497/07-10-2014 projects.

\section{References}

[1] K. Freese, Review of Observational Evidence for Dark Matter in the Universe and in upcoming searches for Dark Stars, EAS Publ.Ser. 36, 113 (2009) arXiv:0812.4005 [astro-ph].

[2] J. Alexander et. al., Dark Sectors 2016 Workshop: Community Report, (2016) arXiv:1608.08632 [hep-ph].

[3] O. Adriani et al., [PAMELA Collaboration], An anomalous positron abundance in cosmic rays with energies 1.5-100 GeV, Nature 458, 607 (2009).

[4] T. Blum et al., Calculation of the Hadronic Vacuum Polarization Contribution to the Muon Anomalous Magnetic Moment Phys. Rev. Lett. 121, 022003 (2018).

[5] M. Raggi and V. Kozhuharov, Proposal to search for a dark photon in positron on target collisions at DAФNE linac, Adv. High Energy Phys. 509, (2014) 959802.

[6] B. Buonuomo, G. Mazzitelli and P. Valente, Performance and upgrade of the.DAФNE Beam Test Facility (BTF), Adv. High Energy Phys. 509, (2014) 959802 IEEE Transactions on Nuclear Science 52(4), 824 (2005).

[7] F.Oliva, on behalf of the PADME Collaboration, Operation and performance of the active target of $P A D M E$, in press NIM A (2019).

[8] S. Ivanov and V. Kozhuharov, The Charged Particle Veto System of the PADME Experiment AIP Conference Proceedings 2075, 080005 (2019).

[9] G. Piperno, on behalf of the PADME Collaboration, The PADME electromagnetic calorimeter, J. Phys. Conf. Ser. 1162012031 (2019).

[10] M. Raggi et al., Performance of the PADME calorimeter prototype at the DAFNE BTF, NIM A 862, 31 (2017). 